\title{
The dynamics of an extremely young cluster probed for the first time
}

\author{
Christine Ducourant, ${ }^{1,2}$ Sylvain Bontemps, ${ }^{1,2}$ Didier Despois, ${ }^{1,2}$ \\ Philippe André, ${ }^{3}$ Ramachrisna Teixeira, ${ }^{4}$ Estelle Moraux,${ }^{5}$ Jerome \\ Bouvier, ${ }^{5}$ Alberto Krone-Martins ${ }^{1,2,4}$ and Dimitris Sinachopoulos ${ }^{6}$ \\ ${ }^{1}$ Université de Bordeaux, Observatoire Aquitain des Sciences de l'Univers, \\ 2 rue de l'Observatoire, BP 89, F-33271 Floirac Cedex, France \\ ${ }^{2}$ CNRS, UMR 5804, Laboratoire d'Astrophysique de Bordeaux, 2 rue de l'Observatoire, BP 89, \\ F-33271 Floirac Cedex, France \\ email: ducourant@obs.u-bordeaux1.fr \\ ${ }^{3}$ CEA, DSM, IRFU, Service d'Astrophysique, 91191 Gif-sur-Yvette, France; Laboratoire AIM, \\ CEA/DSM - CNRS - Université Paris Diderot, IRFU/Service d'Astrophysique, CEA-Saclay, \\ 91191 Gif-sur-Yvette, France, \\ ${ }^{4}$ Instituto de Astronomia, Geofísica e Ciências Atmosféricas, Universidade de São Paulo, \\ Rua do Matão 1226, Cidade Universitária, São Paulo, Brazil \\ ${ }^{5}$ Laboratoire d'Astrophysique de l'Observatoire de Grenoble, 414, Rue de la Piscine, \\ Domaine Universitaire, BP 53, 38041 Grenoble Cedex 09, France \\ ${ }^{6}$ National Observatory of Athens, P. O. Box 20048, Thissio 11810, Athens, Greece
}

\begin{abstract}
We present a proper-motion program that we have developed at ESO to measure, for the first time, proper motions of members of the nearby $(125 \mathrm{pc})$, very young $\left(4 \times 10^{5} \mathrm{yr}\right)$ infrared $\rho$ Ophiuchi cluster. Repeated imaging over an extended period will allow us to measure the global proper motion of the cluster and its velocity dispersion and will provide access to the $\sim 1 \mathrm{~km} \mathrm{~s}^{-1}$ velocities for objects as faint as $K=15.7 \mathrm{mag}$. Access to the velocity field of such an extremely young cluster is of crucial importance to investigate the origin and early evolution of open cluster systems.
\end{abstract}

Keywords. stars: distances, stars: kinematics, stars: evolution, stars: formation

\section{Introduction}

Stars are believed to form predominantly in associations, and young stars are observed in infrared (IR) clusters still embedded in nearby molecular clouds (e.g., Lada et al. 1991; André et al. 2007). These extremely young clusters (1-2 Myr) appear to be the precursors of the visible open clusters and/or of dispersed Galactic stellar streams. While OB associations have recently been surveyed by Hipparcos for detailed proper-motion studies (de Zeeuw et al. 1999), no IR clusters have yet been studied in this sense.

Studying proper motions in IR clusters is the only way to determine if these clusters are gravitationally bound and therefore stable, or unbound, i.e., already in the process of dispersing. Indeed, embedded clusters are young enough to be still associated with a large amount of molecular gas, which may keep the system of young stars and gas still bound. Proper-motion investigation is also an excellent and classical way to significantly improve the completeness of the known cluster members and can be applied to IR clusters. They are young enough to not yet have lost any stars and therefore constitute excellent cases to investigate the fundamental issue of the shape and origin of the initial mass function $(\mathrm{IMF})$. 
For these reasons, searching for proper motions in embedded IR clusters is unique and should shed light on two crucial issues in star-formation studies, i.e., the origin/early evolution of young stellar clusters and the IMF observed directly at the stellar birth place.

The $\rho$ Ophiuchi IR cluster is unique in its proximity $(125 \mathrm{pc})$, its richness in young stars and protostars, and its extreme youth (the youngest cluster known, $\sim 0.5 \mathrm{Myr}$ old; André et al. 2007). It is certainly the best target presently accessible from the ground to measure, for the first time, internal stellar motions in an embedded IR cluster. In addition, we can use various surveys that have studied this cluster (Bontemps et al. 1998; Duchêne et al. 2004; Stamatellos et al. 2007), which have achieved a census of the population of young stars with IR excesses. This allowed these authors to discuss, for the first time, the mass function in such a young cluster down to masses of less than $0.1 \mathrm{M}_{\odot}$.

\section{The project}

\subsection{Selection of the field}

We have selected (from Bontemps et al. 1998) the subcluster $\rho$ Ophiuchi F, which exhibits a clear association of young stars with the Ophiuchi F molecular dense core (see Motte et al. 1998; André et al. 2007). This dense core/IR cluster is roughly a 0.1 pc-radius sphere containing $15-30 \mathrm{M}_{\odot}$ (Motte et al. 1998) of gas and $5-10 \mathrm{M}_{\odot}$ of stars. The typical Keplerian velocity in such a system is then $v_{\mathrm{K}}=\left(2 G M_{\mathrm{tot}} / R\right)^{1 / 2}$, or 1.3 and $1.7 \mathrm{~km}$ $\mathrm{s}^{-1}$ for, respectively, 20 and $40 \mathrm{M}_{\odot}$ spheres with a radius of $R=0.1 \mathrm{pc}$. The typical

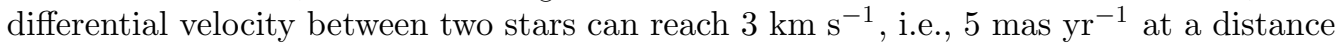
of $125 \mathrm{pc}$. The systematic proper motion of the $\rho$ Ophiuchi IR cluster can be estimated from the proper motion of the associated, nearby Upper Scorpius OB association recently measured by de Zeeuw et al. (1999) with Hipparcos. They found a global proper motion

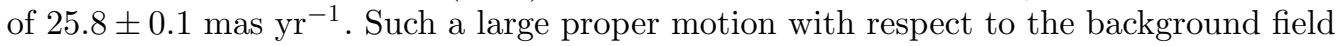
stars can easily be measured with our observational technique. We plan to investigate the proper motion of stars down to $K_{\mathrm{s}}=15.7$ ( images), which corresponds to a $0.02 \mathrm{~L}_{\odot}\left(\right.$ for $A_{\mathrm{V}}=30$ ), or $0.045 \mathrm{M}_{\odot}$ i.e., a young brown dwarf. We will be able to recognize all members, irrespective of their nature (protostars, young stars with or without IR excess, brown dwarfs). Our completeness will therefore be limited only by our ability to recognize systematic proper motions of the members (which, in principle, is not significantly flux dependent). This is extremely important for addressing the issue of the shape and origin of the IMF in this cluster, since we will get a membership census based on an unbiased technique. Additional spectroscopic observations of kinematic members will confirm their nature.

\subsection{Binarity}

We have investigated, through Monte Carlo simulations, the effects of binarity. The data for the simulation were taken from Bontemps et al. (1998; IMF), D'Antona \& Mazzitelli (1994; $L-M$ relation), Duquennoy \& Mayor (1991; binary-period distribution). Orbital inclinations were taken as random and component masses considered uncorrelated. Several thousand systems were simulated and analysed in terms of proper motion. We normalize the results, for presentation purposes, to 100 binary systems (assuming that all young stars belong to binary systems). The simulation results in 65 unresolved and 35 resolved couples for 0.7 arcsec seeing. We thus have 135 'objects' (65 unresolved binaries and $2 \times 35$ resolved-system components). Only nine unresolved binaries have a proper motion

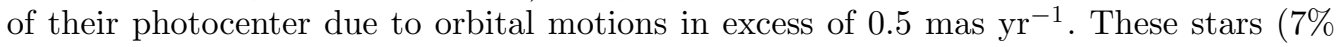
of the simulated population) will affect the results slightly. Twenty six components of 


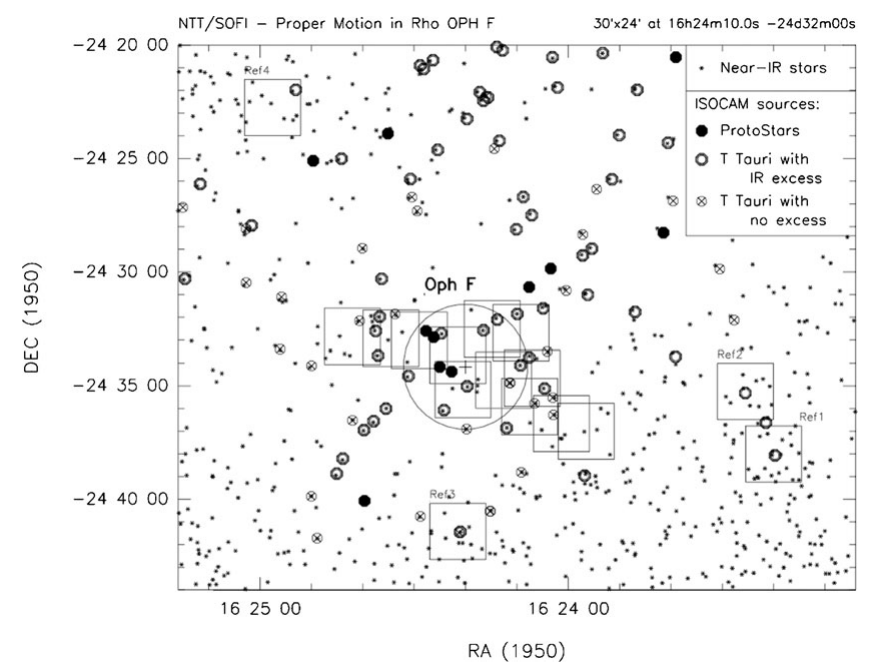

Figure 1. NTT/SOFI selected fields in the $\rho$ Ophiuchi E-F dark core.

resolved binary systems also exhibit a measurable proper motion due to orbital motion. However, the separation of these 26 binaries with proper motions $\mu>0.5$ mas $\mathrm{yr}^{-1}$ appears to be very small and essentially always less than 2 arcsec. Since the typical separation of young stars is much greater than $2 \operatorname{arcsec}$ (on the order of 1 arcmin), this ensures that we will always recognize these pairs. They will be put apart for special analysis. It is therefore clear that binarity does not significantly affect our first goal, determination of the internal velocity dispersion of the forming cluster. It does not hamper either our second important goal, i.e., using proper motion as a cluster-membership criterion. This motion is much larger than any orbital motions and internal velocity dispersion (25 mas $\mathrm{yr}^{-1}$; from Hipparcos).

\subsection{Astrometry}

The astrometric objective of this program is to measure individual proper motions with

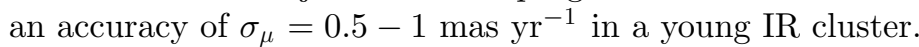

The first observational epochs were acquired in April 2001, 2002, 2003 and 2004. Figure 1 presents the observed dense core with the 10 overlapping fields. Data have been measured and reduced using software developed in Bordeaux (see Ducourant et al. 2007). We have derived preliminary proper motions for 89 stars in the zone covered by our ob-

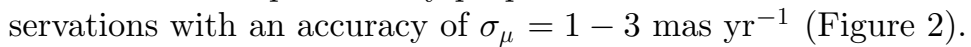

The known problem of field distortions of the SOFI instrument had to be addressed. Much effort was dedicated to correct our measurements for these distortions. To minimize residual effects, we also included (in the global reduction) photometric images (SOFI large field) that suffer from less distortion, to average the effects.

The combined problem of field distortion, which has a significant impact on the astrometry, and the poor numbers of stars present in each field led us to extend the program, acquiring a new set of observations in May 2009. With the addition of an additional epoch 8 years after the first observations, the astrometric precision should be improved

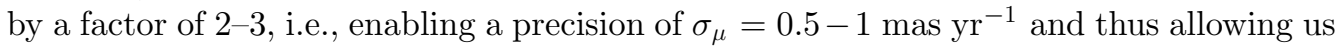
to measure the largest expected differential velocities within the cloud and the expected atypical velocities greater than that of the cloud (due to, e.g., binary ejection, ...). Data are being reduced and will be presented soon. 


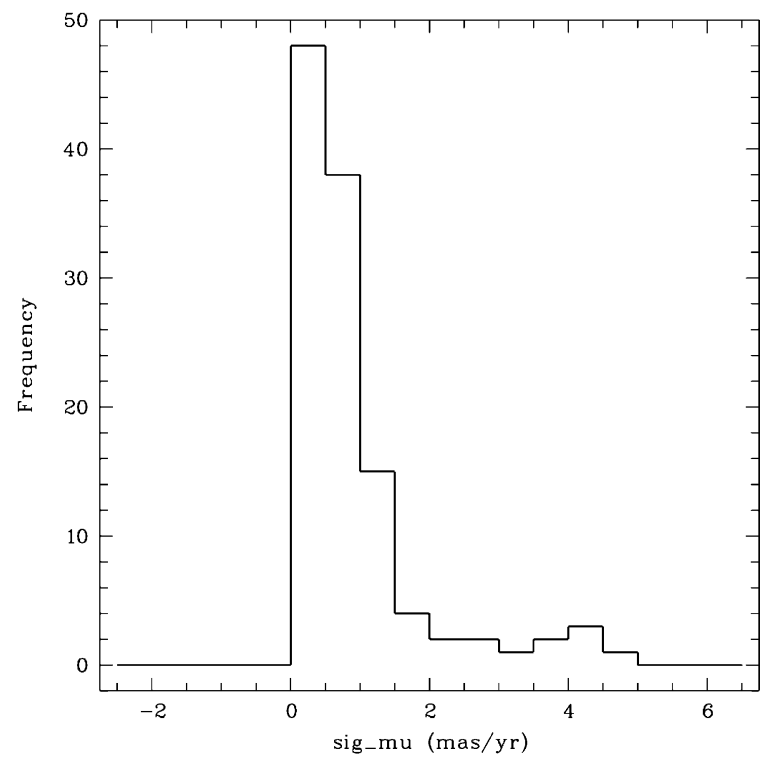

Figure 2. Histogram of the precisions of proper motions $\sigma_{\mu}$ derived from a preliminary global overlap solution of the 10 overlapping fields including the first four observational epochs.

\section{References}

André, P., Belloche, A., Motte, F., \& Peretto, N. 2007, A\& A, 472, 519

André, P., Bouwman, J., Belloche, A., \& Hennebelle, P. 2004, ApSS, 292, 325

Barsony, M., Ressler, M. E., \& Marsh, K. A. 2005, ApJ, 630, 381

Bontemps, S., et al. 1998, in: J. Yun \& R. Liseau (eds.), Star Formation with the Infrared Space Observatory, ASP Conf. Ser., 132, p. 141

D'Antona, F. \& Mazzitelli, I. 1994, ApJS, 90, 467

Duchêne, G., Bouvier, J., Bontemps, S., André, P., \& Motte, F. 2004, A\&\&A, 427, 651

Ducourant, C., Teixeira, R., Chauvin, G., Daigne, G., Le Campion, J.-F., Song, I., \& Zuckerman, B. $2008, A \mathscr{E} A$ (Letters), 477, L1,

Ducourant, C., Teixeira, R., Hambly, N. C., Oppenheimer, B. R., Hawkins, M. R. S., Rapaport, M., Modolo, J., \& Lecampion, J. F. 2007, A\& $A$, 470, 387

Duquennoy, A. \& Mayor, M. 1991, A\&A A, 248, 485

Lada, E. A., Bally, J., \& Stark, A. A. 1991, ApJ, 368, 432

Lazorenko, P. F., Mayor, M., Dominik, M., Pepe, F., Segransan, D., \& Udry, S. 2007, A $6 A$, 471, 1057

Stamatellos, D., Ward-Thompson, D., Whitworth, A. P., \& Bontemps, S. 2007, A\&SA, 462, 677

Teixeira, R., Ducourant, C., Chauvin, G., Krone-Martins, A., Song, I., \& Zuckerman, B. 2008, $A \& A, 489,825$

de Zeeuw. P. T., Hoogerwerf, R., de Bruijne, J. H. J., Brown, A. G. A., \& Blaauw, A. 1999, AJ, 117,354 